Voix et Images

voixetimages

\title{
Québécoise à vendre ou la revanche de Petit Canada
}

\section{Jonathan M. Weiss}

Volume 6, numéro 1, automne 1980

Gilles Marcotte

URI : https://id.erudit.org/iderudit/200257ar

DOI : https://doi.org/10.7202/200257ar

Aller au sommaire du numéro

Éditeur(s)

Les Presses de l'Université du Québec

ISSN

0318-9201 (imprimé)

1705-933X (numérique)

Découvrir la revue

Citer ce compte rendu

Weiss, J. M. (1980). Compte rendu de [Québécoise à vendre ou la revanche de Petit Canada]. Voix et Images, 6(1), 155-157. https://doi.org/10.7202/200257ar d'utilisation que vous pouvez consulter en ligne.

https://apropos.erudit.org/fr/usagers/politique-dutilisation/ 


\section{Québécoise à vendre ou la revanche de Petit Canada}

pièce écrite et réalisée par Jocelyne Saint-Denis et Ginette Candotti-Besson. en tournée en France

par Jonathan M. Weiss

Quand on pense au théâtre québécois en France, viennent surtout à l'esprit des pièces qui, comme Les Belles-S æurs, Citrouille ou Quatre à quatre, ont été composées au Québec pour un public québécois et ont traversé l'Atlantique avec plus ou moins de succès. Pour la première fois, à ma connaissance, une pièce de théâtre québécoise est écrite et jouée en fonction d'un public français - interdite, pour ainsi dire, à l'importation.

La pièce de Jocelyne Saint-Denis et Ginette Candotti-Besson tient d'une gageure: montrer au public français les relations assez complexes qui existent entre les Québécois et les Français. Voici trois ans que Jocelyne Saint-Denis, ancienne étudiante de l'École Nationale de Théâtre, quittait Montréal et s'installait en France pour perfectionner son double métier d'écrivain et d'actrice. Elle rencontre une Française, Ginette Candotti-Besson, et ensemble elles écrivent une pièce qui, d'une façon résolument antiréaliste et symbolique, raconte la rencontre de deux personnes, de deux cultures. Infatigables, les auteurs sont à la fois interprètes et metteurs en scène de leur pièce qu'elles présentent dans plusieurs villes françaises et au Festival d'Avignon en 1979. Précisons que j'ai vu cette pièce à Toulouse, en mars 1980, devant un public assez jeune et qui comptait au moins deux Canadiens-français.

Mais comment dire aux Français ce qu'un Québécois ou une Québécoise peuvent ressentir en France? Car il faut se garder des généralisations hâtives : ce n'est pas parce qu'Antonine Maillet reçoit le Goncourt ou que le dernier roman de Michel Tremblay est apprécié sur France-Culture que les Français, sauf une petite minorité d'intellectuels, connaissent le Canada français autrement que sous la forme d'un folklore intéressant mais, somme toute, désuet. Entre ceux qui trouvent l'accent rigolo (mais combien charmant!) et ceux pour qui le Québec n'est qu'un rappel de la bonne vie paysanne en Vendée (ou Normandie ou Picardie), qu'y a-t-il à choisir?

Pourtant le Québec conserve un aspect fascinant. C'est cette fascination, aussi bien que celle que la France exerce sur certains Québécois, que 
Jocelyne Saint-Denis et Ginette Candotti-Besson essaient d'analyser. II semble que du côté québécois, l'attrait de la France soit d'abord d'ordre linguistique. Le personnage principal de Québécoise à vendre, une jeune Montréalaise nommée Artémise Rinfret, trimbale jusque dans son lit un énorme Larousse d'il y a cinquante ans; si le verbe «toffer" n'y figure pas, c'est qu'il y a erreur chez les éditeurs français. Mais il y a un lapsus autrement plus grave : le Frère André, objet des affections spirituelles et charnelles d'Artémise, n'a pas son article dans la partie encyclopédique. Lacune qu'Artémise n'hésiterait pas à combler, si seulement elle pouvait venir a Paris... Un concours d'essai qui n'en est pas un (et qui permet ì Artémise de faire connaissance avec le mot quiproquo) fournit l'occasion du voyage tant espéré. En effet, le redoutable Monsieur Larousse, devant la (si séduisante) naïveté de l'essai de la jeune québécoise, va l'inviter chez lui pour l'offrir en cadeau à sa fille (comme jadis on avait fait de Petit Canada).

Portant sa ceinture fléchée, sa tuque, son chandail bleu-blanc-rouge du Canadien, Artémise arrive à Paris. Il s'agit ici non pas de faire rire d'une Québécoise assez "quétaine", mais de donner visuellement l'image que les Français se forment des Québécois. Détail expressionniste, ce costume sera le prétexte d'un des moments les plus réussis de la pièce où Artémise raconte des scènes de mort et d'agonie vues (ou imaginées) par elle dans sa jeunesse, en compagnie d'une Française qui se dilate la rate de rire à son accent. Comme pour le costume, c'est l'apparence seule qui compte. Ironiquement, l'intérêt que portent les Français à la Québécoise est proportionnel à leur incompréhension de son parler.

Chez Monsieur Larousse et sa fille Marie-France, les dictionnaires sont plus modernes (et plus petits) qu'à Montréal mais c'est un détail trompeur, car le vocabulaire en France offre des difficultés qu'Artémise ne soupçonnait pas. En effet, comment faire comprendre que la même chose, de l'autre côté de l'océan, porte un autre nom? Mais il y a plus grave. Invitée au lycée par Marie-France, qui y poursuit ses études, Artémise est sévèrement mise à l'épreuve par le professeur qui lui colle zéro sur zéro. Pauvre Artémise, avec ses trois petits livres pieux rapportés du Québec à côté des cinq beaux tomes de l'Histoire de France que possède Marie-France. Interrogée sur l'histoire de son pays ("Pourquoi parlez-vous français, Mademoiselle?»), Artémise, ne sachant que répondre, balbutie «mes parents parlent français, alors moi aussi...".

Marie-France prend la relève en récitant mot pour mot l'histoire du Canada telle qu'elle est contenue dans un de ses beaux livres. Pour elle, l'histoire est une série de mots à apprendre; pour Artémise, l'histoire est avant tout vécue, et celle de son pays reste à faire.

Mais elle aura sa revanche, cette Québécoise toujours soucieuse de faire entrer le Frère André dans le dictionnaire Larousse (canonisation plus importante que celle que confère le Pape). Et, détail significatif, c'est le théâtre qui lui offrira le moyen de se venger des Larousse, père, fille et dic- 
tionnaire. Le professeur, dans un élan de générosité, donne à Artémise une dernière chance de se rattraper; il s'agira de composer une scène et de la faire jouer devant la classe. Artémise n'hésite point; elle décide de monter la vie et le massacre d'un saint martyr canadien. Malgré une hésitation bien compréhensible, Marie-France jouera le rôle du martyr, mais au fur et à mesure que la répétition de la scène avance, Artémise cessera d'incarner la voix de Dieu pour devenir, avec une férocité surprenante. I'Iroquois en colère. Elle finira par s'identifier complètement aux Indiens. Délaissant son français pour l'algonquin, elle saisira Marie-France à la gorge et, au moment où la pièce se termine, l'abattra avec un fusil. Petit Canada aura été vengé.

II faut souligner l'originalité de la fin de cette pièce. Si le début nous montrait des réactions personnelles, nous passons, dans la deuxième partie à des considérations d'ordre mythique. II a fallu qu'Artémise se débarrasse définitivement de son dictionnaire et de tous les Larousse pour comprendre qu'elle n'était pas, en fin de compte, une Française de deuxième ordre.

En s'identifiant aux opprimés plutôt qu'aux colonisateurs, elle a fait un pas en avant dans la revendication de sa propre identité.

Pièce agressive, Québécoise à vendre aura du moins provoqué une prise de conscience chez certains Français; chez d'autres, pour qui les allusions au Frère André et aux saints martyrs, de même que l'emploi d'un vocabulaire bien québécois poseront des problèmes de compréhension, la pièce aura moins réussi.

Jocelyne Saint-Denis songe, à son retour à Montréal, à en faire une adaptation pour le public québécois. Reste à voir si Québécoise à vendre pièce sérieuse et provocatrice, passera la rampe à Montréal. 